This item was submitted to Loughborough's Research Repository by the author.

Items in Figshare are protected by copyright, with all rights reserved, unless otherwise indicated.

\title{
Health monitoring system for transmission shafts based on adaptive parameter identification
}

PLEASE CITE THE PUBLISHED VERSION

https://doi.org/10.1016/j.ymssp.2017.11.023

\section{PUBLISHER}

(C) Elsevier

VERSION

AM (Accepted Manuscript)

\section{PUBLISHER STATEMENT}

This work is made available according to the conditions of the Creative Commons Attribution-NonCommercialNoDerivatives 4.0 International (CC BY-NC-ND 4.0) licence. Full details of this licence are available at: https://creativecommons.org/licenses/by-nc-nd/4.0/

\section{LICENCE}

CC BY-NC-ND 4.0

\section{REPOSITORY RECORD}

Souflas, loannis, Antonios Pezouvanis, and Kambiz Morteza Ebrahimi. 2018. "Health Monitoring System for Transmission Shafts Based on Adaptive Parameter Identification”. figshare. https://hdl.handle.net/2134/33361. 


\title{
Health Monitoring System for Engine Dynamometer Shafts Based on Adaptive Parameter Identification
}

\author{
Ioannis Souflas. Antonios Pezouvanis. Kambiz M. Ebrahimi* . \\ Department of Aeronautical and Automotive Engineering, Loughborough University, \\ Loughborough, UK
}

\begin{abstract}
A health monitoring system for engine dynamometer shafts is proposed. The solution is based on the real-time identification of the physical characteristics of the coupling shaft i.e. stiffness and damping coefficients, by using a physical oriented model and linear recursive identification. The efficacy of the suggested condition monitoring system is demonstrated on a prototype transient engine testing facility equipped with a coupling shaft capable of varying its physical properties. Simulation studies reveal that coupling shaft faults can be detected and isolated using the proposed condition monitoring system. Besides, the performance of various recursive identification algorithms is addressed. The results of this work recommend that the health status of engine dynamometer shafts can be monitored using a simple lumped-parameter shaft model and a linear recursive identification algorithm which makes the concept practically viable.
\end{abstract}

\footnotetext{
* Corresponding Author

Kambiz M. Ebrahimi, Department of Aeronautical and Automotive Engineering, Loughborough

University, Loughborough, UK

Email: K.Ebrahimi@lboro.ac.uk
} 


\section{Keywords}

Health Monitoring; Kalman Filter; Shaft; Engine; Dynamometer

\section{Introduction}

Coupling shafts are responsible for transferring kinetic energy between rotating components and machines. Applications can be found in different engineering areas such as power generation plants, machine tool spindles, robotics, marine, aeronautical and automotive propulsion systems. Depending on the purpose of the application, coupling shafts can be designed with different characteristics to satisfy the needs of the user. One of the absolute minimum requirements for every application is the safe and reliable operation of the shaft. Therefore, it is of major importance to be able to monitor the health status of rotor shafts to avoid catastrophic damages.

The condition monitoring of coupling shafts is a well investigated area, and as a result numerous reliable solutions can be found in literature. More specifically, the effectiveness of signal-based techniques using vibration measurements and spectrum or wavelet analysis has been reported in several studies (Cade et al. 2005; Tlaisi et al. 2012; Xiang et al. 2008; Chaubey et al. 2014). Various model-based approaches have also been presented, Castejón et al. (Castejón et al. 2015) presented the use of artificial neural networks and multiresolution analysis for the automatic detection of rotor cracks. Sekhar (Sekhar 2004) showed an online model-based condition monitoring approach based on a rotor-bearing system redundant model using a Finite Element Model (FEM). The combination of FEMs and online system identification procedures has also been proven to be an effective tool for the detection of shaft damages (Seibold \& Fritzen 1995; 
Seibold \& Weinert 1996). State of the art fault detection techniques are based on the identification of parameters with physical interpretation that can be found commonly in FEMs using various type of the Kalman filters (Yang et al. 2006; Hsieh et al. 2013; Wang et al. 2014; Calabrese et al. 2016).

This paper also focuses on the use of physically oriented models and Kalman filtering for the health monitoring of engine dynamometer shafts. However, the proposed coupling shaft health monitoring methodology is significantly simpler than the previously reported approaches. In particular, instead of using FEMs and non-linear recursive identification algorithm e.g. Extended Kalman Filter (EKF), a simple lumped-parameter coupling shaft model and a linear recursive identification algorithm are employed. This makes the monitoring system practically viable and more robust. Additionally, it is important to highlight that the proposed solution does not require any model training exercise compared to FEMs where experiments are need prior to the application to parameterise the models.

As aforementioned the proposed method is based on the on-line identification of two physical characteristics i.e. stiffness and damping coefficients, of a coupling using measurements that can be found commonly in industrial engine testing facilities. More specifically, these measurements are the speed and position of the dynamometer and engine separately, and the torque acting on the shaft. A schematic overview of the suggested monitoring system is presented in Fig. 1. 


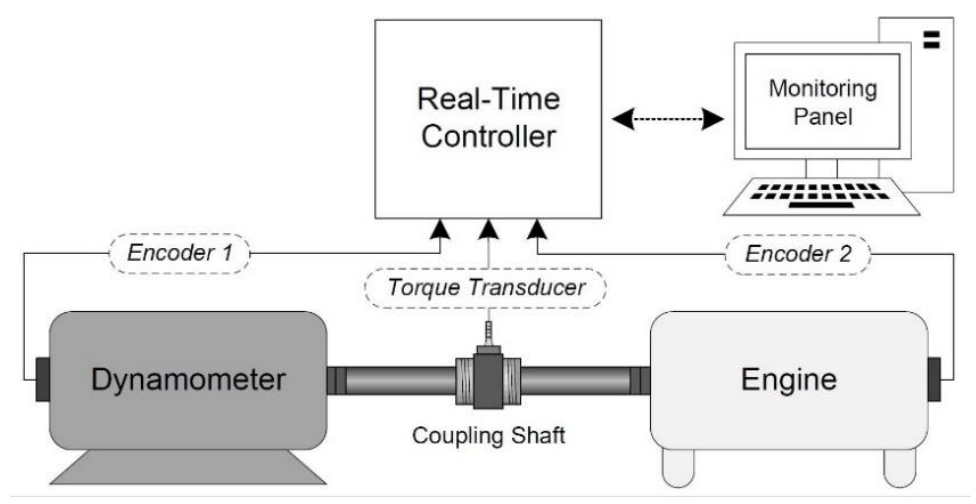

Figure 1: Conceptual diagram of the engine dynamometer shaft health monitoring system.

The paper is arranged as follows. Firstly, a lumped-parameter dynamic model of a transient engine testing facility is derived. Secondly, candidate adaptive parameter identifications algorithms are introduced. Thirdly, details on the practical implementation of the suggested condition monitoring system are discussed. Then, experimental and simulation results are presented, verifying the functionality of the proposed shaft health monitoring system. Finally, based on the faced challenges and the results of this work, some conclusions and potential future directions are outlined. 


\section{Theoretical Principles}

The theoretical preliminaries that are required for the successful practical implementation of the proposed system are discussed in this section.

\section{Engine Dynamometer Shaft Modelling}

The suggested on-line condition monitoring system is based on a simple engine dynamometer shaft lumped-parameter model and the continuous tracking of its physical parameters using recursive identification algorithms. Considering the engine dynamometer shaft as a two degree of freedom rotating system, the free body diagram is drawn as in Fig. 2.

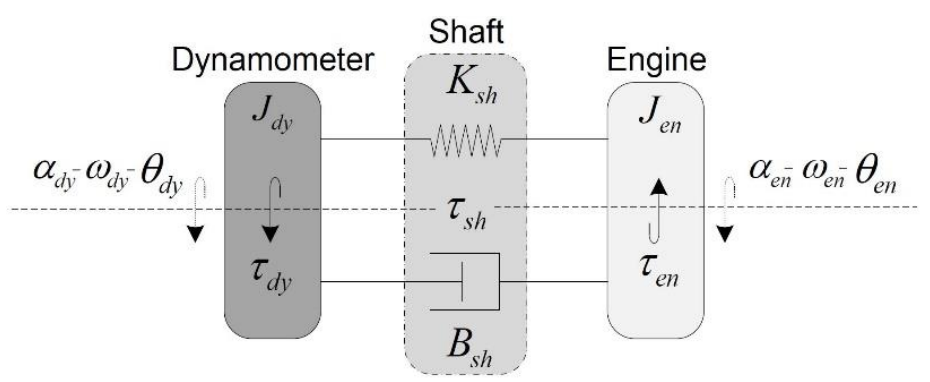

Figure 2: Free body diagram (2-DOF) of the engine dynamometer shaft system.

Assuming that the dynamometer is the primary source of the system and that the moment of inertia of the shaft is smaller than the moment of inertia of the dynamometer and the engine (Szabat et al. 2011), the principle governing equations of the system can be expressed using state-space formalism.

$$
\begin{aligned}
& \dot{x}(t)=A x(t)+B u(t) \\
& y(t)=C x(t)
\end{aligned}
$$


here, system's output $(y)$, inputs $(u)$ and states $(x)$ are:

$$
y=\tau_{s h}, \quad u=\left[\begin{array}{ll}
\tau_{d y} & \tau_{e n}
\end{array}\right], \quad x=\left[\begin{array}{lllll}
\tau_{d y} & \theta_{d y} & \omega_{d y} & \theta_{e n} & \omega_{e n}
\end{array}\right]^{T}
$$

whilst, the parameters of the state-space coefficients are given bellow:

$$
A=\left[\begin{array}{ccccc}
0 & K_{s h} & B_{s h} & -K_{s h} & -B_{s h} \\
0 & 0 & 1 & 0 & 0 \\
0 & -\frac{K_{s h}}{J_{d y}} & 0 & \frac{K_{s h}}{J_{d y}} & \frac{B_{s h}}{J_{d y}} \\
0 & 0 & 0 & 0 & 1 \\
0 & \frac{K_{s h}}{J_{e n}} & \frac{B_{s h}}{J_{e n}} & -\frac{B_{s h}}{J_{e n}} & 0
\end{array}\right], \quad B=\left[\begin{array}{cc}
0 & 0 \\
0 & 0 \\
\frac{1}{J_{d y}} & 0 \\
0 & 0 \\
0 & -\frac{1}{J_{e n}}
\end{array}\right], \quad C=\left[\begin{array}{lllll}
1 & 0 & 0 & 0 & 0
\end{array}\right]
$$

where, the symbols $\tau, \omega, \theta, J, B$ and $K$ represent the torque, angular velocity, angular position, moment of inertia, the damping coefficient and the stiffness coefficient respectively, while the subscripts $d y$, en and sh indicate the dynamometer, the engine and the shaft accordingly.

For more information regarding the mathematical formulation of the overall system model refer to (Souflas et al. 2014; Souflas et al. 2015).

According to the above system, the torque of the shaft can be rewritten as follows:

$$
\tau_{s h}(t)=B_{s h}\left[\omega_{d y}(t)-\omega_{e n}(t)\right]+K_{s h}\left[\theta_{d y}(t)-\theta_{e n}(t)\right]
$$

The mathematical structure of the physical model of the coupling shaft (Eq. 2) is equivalent to the standard format of linear-in-the-parameter static systems (Åström \& Eykhoff 1971).

$$
y(t)=\varphi^{T}(t) \vartheta+\epsilon(t)
$$




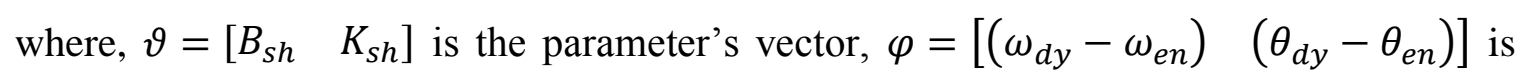
the vector of independent variables, $y=\left[\tau_{s h}\right]$ is the dependent variable vector, and finally $\epsilon$ is a random error term.

The above statement indicates that the physical parameters of the coupling shaft model i.e. stiffness and damping coefficients, could be tracked in real-time by implementing linear recursive identification algorithms. In this work, the effectiveness of three different linear recursive identification algorithms is assessed. Details on the algorithms that were employed in this work are presented on the following section. 


\section{Recursive Identification Algorithms}

The key characteristic of recursive parameter identification techniques is that they allow the estimation of model parameters in parallel to the operation of the real system. Suppose there exist an initial estimate $\hat{\vartheta}_{t-1}$ at iteration $t-1$, then recursive identification algorithms aim to compute a new estimate $\hat{\vartheta}_{t}$ by modifying $\hat{\vartheta}_{t-1}$ when a new observation becomes available at iteration $t$. There is indeed a substantial literature on recursive estimation theory, for general reading refer to (Ljung \& T. Söderström 1983; Ljung 2002; Åström \& Wittenmark 2013).

Linear recursive identification algorithms are derived from the conventional Kalman filter that was initially developed for state estimation of linear time invariant systems.

$$
\begin{aligned}
& K(t)=A P(t) C^{T}\left[C P(t) C^{T}+R_{2}\right]^{-1} \\
& P(t+1)=R_{1}+A P(t) A^{T}-K(t) C P(t) C^{T} K^{T}(t) \\
& \hat{x}(t+1)=A \hat{x}(t)+B u(t)+K(t)[y(t)-C \hat{x}(t)]
\end{aligned}
$$

where, $\hat{x}(t)$ is the state estimates, $K(t)$ is the Kalman gain, and $P(t)$ is the covariance matrix of the state estimates.

The Kalman filter (Eq. 4) can also be used for the estimation of parameters of a linear regression problem (e.g. Eq. 3) by setting,

$$
\hat{x}(t)=\hat{\vartheta}(t) \quad A=I \quad B=0 \quad C=\varphi(t)
$$

This allows the formulation of a general optimal tracking recursive algorithm for linearin-the-parameter problems as follows (Eq. 5): 


$$
\begin{aligned}
& K(t)=P(t) \varphi(t)\left[\varphi(t) P(t) \varphi^{T}(t)+R_{2}\right]^{-1} \\
& P(t+1)=R_{1}+P(t)-K(t) \varphi^{T}(t) P(t) \\
& \hat{\vartheta}(t+1)=\hat{\vartheta}(t)+K(t)\left[y(t)-\varphi^{T}(t) \hat{\vartheta}(t)\right]
\end{aligned}
$$

Notice that for the execution of the above recursive algorithm is required to have an initial estimate of the model parameters $\left(\hat{\vartheta}(0)=\vartheta_{0}\right)$ and the covariance matrix $(P(0)=$ $P_{0}$ ). Furthermore, the values of the observation covariance $R_{1}$ and the parameter/process covariance matrix $R_{2}$ need to be carefully selected in order to track the parameter estimates effectively. For instance, the appropriate selection of these matrices would enable the identification of systems with sudden changes and time-varying parameters. However, these values are rarely known explicitly to the user, hence some ad-hoc values are usually chosen. Numerous ad-hoc alternatives can be found in literature, the three different algorithms that were used in this study are discussed in the following paragraphs.

\section{Recursive Least Squares (RLS)}

The standard recursive least squares is a well-known recursive identification algorithm. The principal idea here is the minimisation of the sum of the least squares error between the observed $y(t)=0$ and calculated responses $\varphi^{T}(t) \vartheta(t)$ (Eq. 6) by recursively adjusting the parameters of the model (Ljung 2002).

$$
\min _{\vartheta} \sum_{t=0}^{n} e^{2}(t) \Leftrightarrow \min _{\vartheta} \sum_{t=0}^{n}\left[y(t)-\varphi^{T}(t) \vartheta(t)\right]^{2}
$$


The algorithm is identical to the Kalman filtering approach with the main difference located in the values of the observation covariance $R_{1}$ and the parameter covariance $R_{2}$. In particular, the measurement covariance matrix is assumed to be zero $\left(R_{1}=0\right)$ while the parameter covariance matrix is set equal to one $\left(R_{2}=0\right)$. Under those circumstances, the RLS algorithm is mainly applicable to systems where the parameters are constant i.e. they don't vary overtime. Practically, this means that the standard RLS would not be the best tool for condition monitoring purposes as it would not track possible changes due to system malfunctions. On the other hand, in case where parameter changes are not expected, then it is indeed a very simple and convenient algorithm to use. The RLS algorithm is summarised below:

\section{Algorithm 1 Recursive Least Squares - RLS}

\section{Require: Initialisation}

$$
\begin{aligned}
& \hat{\vartheta}(0)=\vartheta_{0} \\
& P(0)=P_{0}
\end{aligned}
$$

\section{Ensure: Implementation}

$$
\begin{aligned}
& K(t)=P(t) \varphi(t)\left[\varphi(t) P(t) \varphi^{T}(t)+1\right]^{-1} \\
& P(t+1)=P(t)-K(t) \varphi^{T}(t) P(t) \\
& \hat{\vartheta}(t+1)=\hat{\vartheta}(t)+K(t)\left[y(t)-\varphi^{T}(t) \hat{\vartheta}(t)\right]
\end{aligned}
$$

As it can be seen in Algorithm 1 prior to the implementation of the RLS algorithm it is required to have initial estimates of the model parameters $\vartheta_{0}$ and the covariance 
matrix $P_{0}$. These estimates are determined based on the level of the a-priori knowledge of the user about the system.

$$
\hat{\vartheta}(0)=0_{1, p_{t}} \quad P(0)=c I_{p_{t}}
$$

Where the subscript $p_{t}$ is the total number of parameters, $0_{1, p_{t}}$ is a zero matrix (can be set to zero if no prior knowledge exists) with one row and $p_{t}$ number of columns, $I_{p_{t}}$ is an identity matrix of size $p_{t}$, and the parameter $c$ (can be set to 1000 if no prior knowledge exists) represents the confidence in the initial parameter estimates i.e. the more accurate the initial parameter estimates the lower the value of $c$.

\section{Recursive Least Squares with Vector Forgetting Factor (RLSVEF)}

One of the main disadvantages of the standard RLS algorithm is its incapacity to track time-varying system parameters. The so called RLS with Forgetting (RLSF) factor algorithm is another version of the standard RLS that allows the tracking of slowly timevarying parameters. The main difference compared to the standard RLS is located on the least squares minimisation criterion (Ljung 2002):

$$
\min _{\vartheta} \sum_{t=0}^{n} \lambda^{n-t} e^{2}(t) \Leftrightarrow \min _{\vartheta} \sum_{t=0}^{n} \lambda^{n-t}\left[y(t)-\varphi^{T}(t) \vartheta(t)\right]^{2}
$$

where, $\lambda$ is the so called forgetting factor which is exponentially proportional to the number of samples $n$, and $0 \leq \lambda \leq 1$. As a matter of fact $\lambda$ can be either a scalar value, which means that is common for all of the parameters of the model, or a vector $(\Lambda)$, which allows the allocation of multiple forgetting factors in models with multiple parameters. 


$$
\Lambda=\operatorname{diag}\left(\lambda_{1}, \lambda_{2}, \ldots, \lambda_{p_{t}}\right)
$$

Note that the initialisation of the model parameters $\vartheta_{0}$ and the covariance matrix $P_{0}$ is identical to the RLS algorithm, whilst the values of the vector forgetting $\Lambda$ depend on the parameters of the system i.e. time-varying or time-invariant.

\section{Algorithm 2 RLS with Vector Forgetting - RLSVEF}

\section{Require: Initialisation}

$$
\begin{aligned}
& \hat{\vartheta}(0)=\vartheta_{0} \\
& P(0)=P_{0} \\
& 0 \leq \lambda_{i} \leq 1 \\
& \Lambda=\operatorname{diag}\left(\lambda_{1}, \lambda_{2}, \ldots, \lambda_{p_{t}}\right)
\end{aligned}
$$

\section{Ensure: Implementation}

$$
\begin{aligned}
& K(t)=P(t) \varphi(t)\left[\varphi(t) P(t) \varphi^{T}(t)+1\right]^{-1} \\
& P(t+1)=\Lambda^{-1}\left\{P(t)-K(t) \varphi^{T}(t) P(t)\right\} \Lambda^{-1} \\
& \hat{\vartheta}(t+1)=\hat{\vartheta}(t)+K(t)\left[y(t)-\varphi^{T}(t) \hat{\vartheta}(t)\right]
\end{aligned}
$$

\section{Discrete Square Root Filtering in Covariance Form (DSFC)}

One of the main problems of the aforementioned recursive algorithms is related to numerical issues associated with the windup of the covariance matrix $P$ during steady state conditions (no excitation in the input signals). The numerical properties are 
important if the word length is confined or if the changes of input signals become small, as in adaptive control or fault detection applications (Isermann \& Münchhof 2010). In such cases the source of the problem is located in the propagation of the covariance matrix $P$ by means of the Kalman filter equation (Eq. 8) which results in a matrix that is not positive semidefinite. To circumvent this difficulty, a method that propagates the covariance matrix $P$ in a square root form generally known as Discrete Square Root Filtering in Covariance Form (DSFC) was developed (Kaminski et al. 1971). This approach can provide twice the precision of the conventional recursive estimators in illconditioned problems.

$$
P=S S^{T}
$$

where, $S$ is known as the square root of the covariance matrix $P$.

This allows the rearrangement of the conventional recursive least squares formulation which leads to the DSFC algorithm (Algorithm 3). The starting values of the parameter estimates $\hat{\vartheta}(0)$ obey the same rules as the previously reported algorithms. The initialisation of the square root $S(0)$ is an identity matrix multiplied by the square root of a confidence constant i.e. $\sqrt{c} I_{p_{t}}$, where $c$ is the confidence constant, and $p_{t}$ is the total number of model parameters. 
Algorithm 2 RLS with Vector Forgetting - RLSVEF

\section{Require: Initialisation}

$$
\begin{aligned}
& \hat{\vartheta}(0)=\vartheta_{0} \\
& S(0)=S_{0} \\
& 0 \leq \lambda \leq 1
\end{aligned}
$$

Ensure: Implementation

$$
\begin{aligned}
& f(t)=S^{T}(t) \varphi(t) \\
& \psi(t)=\left[f^{T}(t) f(t)+\lambda\right]^{-1} \\
& g(t)=\frac{1}{1+\sqrt{\lambda \psi(t)}} \\
& \gamma(t)=\psi(t) S(t) f(t) \\
& S(t+1)=\frac{S(t)-g(t) \gamma(t) f(t)}{\sqrt{\lambda}} \\
& \hat{\vartheta}(t+1)=\hat{\vartheta}(t)+\gamma(t)\left[y(t)-\varphi^{T}(t) \hat{\vartheta}(t)\right]
\end{aligned}
$$




\section{Practical Implementation}

This section covers the technical features and the implementation details of the health monitoring system.

\section{Test Rig}

The condition monitoring system was developed and tested on a transient engine experimental facility consisting of an electric dynamometer, a single cylinder engine, a shaft with a spider coupling, and the related instrumentation/control system (Fig. 3).

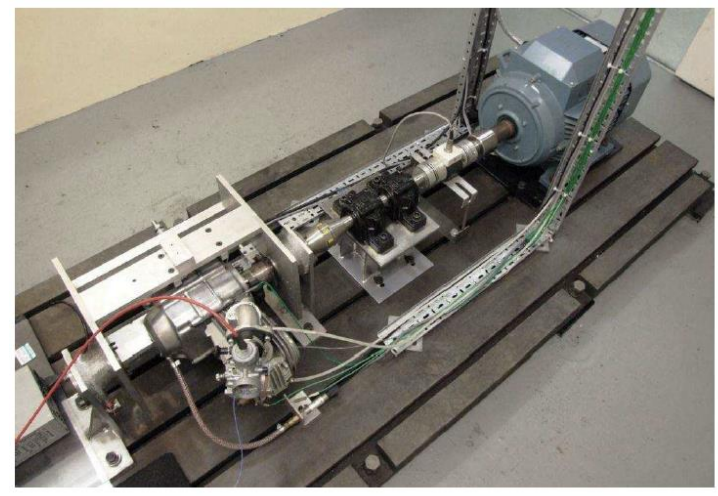

(a)

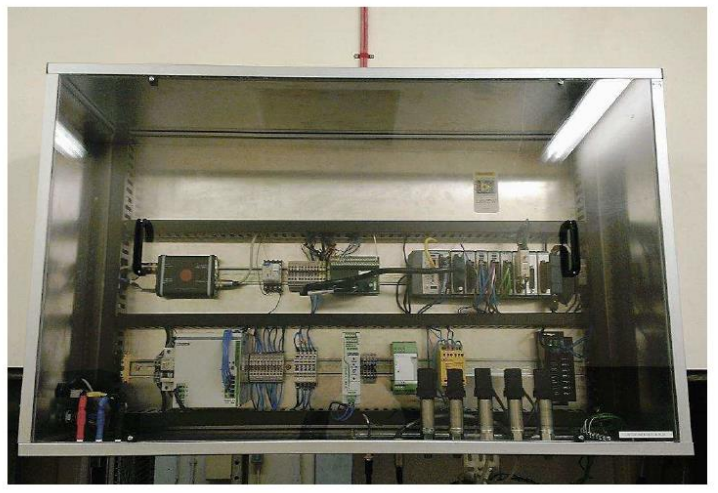

(b)

Figure 3: Transient engine testing facility, (a) engine test bed, (b) instrumentation box.

The physical characteristics of the coupling shaft unit were varied by changing the material of the spider coupling. This allowed the validation of the suggested approach as different spider materials resulted in different stiffness and damping coefficients of the coupling shaft. More specifically, three different spider couplers were employed to test the efficacy of the monitoring system (Fig. 4). Practically speaking, the yellow spider (Fig. 4a) is suitable for low torque systems and have high damping properties, the red 
spider (Fig. 4b) offers medium torque capacity and damping properties, and the green spider (Fig. 4c) offers high torque capacity and minimal damping properties. The actual values of the stiffness and damping coefficients for each spider are tabulated in the Appendix 2, Table 1.

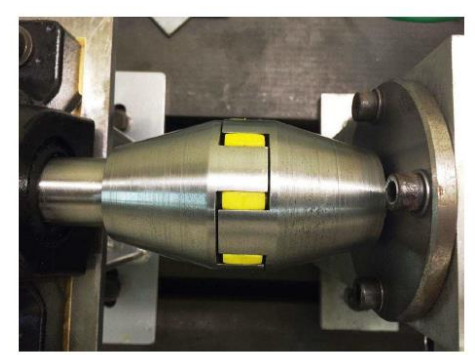

(a)

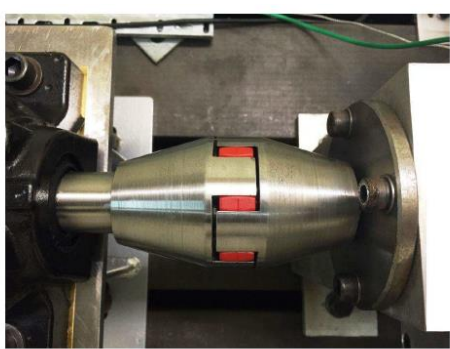

(b)

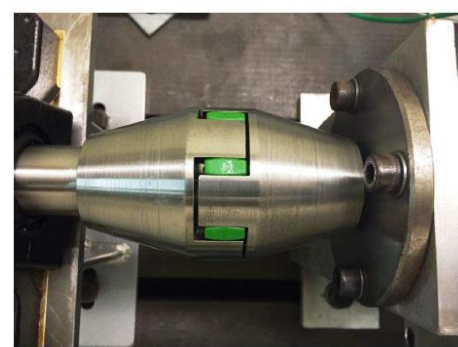

(c)

Figure 4: Coupling shaft spiders with different stiffness and damping characteristics, (a) low torque (yellow), (b) medium torque (red), (c) high torque (green).

\section{Health Monitoring System}

Turning to the instrumentation system, measurements from two quadrature encoders (installed at each end of the coupling shaft i.e. dynamometer and engine) and an in-line torque transducer had to be acquired on a crank-angle basis. This was necessary to ensure sufficient excitation in the measured signal especially during steady state conditions to eliminate windup of the recursive identification algorithm. Consequently for the needs of this study, a custom made high speed data acquisition system was developed using National Instruments hardware (NI-CompactRIO) and software (NI LabVIEW RealTime/FPGA). The sampling frequency $f_{s}$ of the data acquisition systems was determined using Eq. 9: 


$$
f_{s}=\left[\frac{N_{s}}{60} \cdot 360\right] \cdot c_{n y q}
$$

where, $N_{S}$ is the engine speed in RPM, and $c_{n y q}$ is equal to 2 and is used to take into account the Nyquist-Shannon criterion for sufficient sample-rate. Although the measurement signals were all synchronized using the engine encoder (event-based) to ensure equidistant sampling, special handling required to eliminate aliasing effects (Vigild et al. 2000).

The core of the on-line condition monitoring system is a linear recursive estimator that is used to track the physical parameters of the coupling shaft model. The recursive estimator runs in parallel to the operation of the system whilst it makes use of the acquired position, velocity and torque measurements in order to compute the coupling shaft's stiffness and damping coefficients. As is can be seen in Fig. 5 the condition monitoring system consists of two separate modules namely the "Recursive Identification" module and the "Change Detection" module. The inputs to the recursive identification module are the angular position $\delta \theta(t)$ (Eq. 10) and velocity $\delta \omega(t)$ (Eq. 11) differences and the torque of the shaft $\tau_{s h}$.

$$
\begin{gathered}
\delta \theta(t)=\theta_{d y}(t)-\theta_{e n}(t) \\
\delta \omega(t)=\omega_{d y}(t)-\omega_{e n}(t)
\end{gathered}
$$

Before the signals are fed into the recursive identification module they are first filtered using zero-phase distortion filters (forward and backward filtering) to eliminate measurement noises while avoiding phase artefacts. It should be noted that loop 
parallelism is introduced for the filtering task in order to have online processing of the data. After the filtering of the data, the information are used to estimate the parameters of the coupling shaft model i.e. stiffness $\widehat{K}_{s h}(t)$ and damping $\widehat{B}_{s h}(t)$ coefficients, by using linear recursive identification algorithms. For this application the efficacy of three linear recursive identification algorithms, the RLS, the RLSVEF, and the DSFC is addressed. Finally, after the estimation of the parameters the change detection module compares the estimates with true value of the parameters $\left(K_{s h}(t), B_{s h}(t)\right)$ (Table 1) in order to determine the health condition of the coupling shaft. This is achieved simply by calculating the relative error $e(t)$ between the parameter estimates $(\hat{\vartheta}(t))$ and the healthy set of parameters $(\vartheta(t))$.

$$
e(t)=\frac{\vartheta(t)-\hat{\vartheta}(t)}{\vartheta(t)}
$$

Note, that the relative error is calculated independently for each parameter of the coupling shaft i.e. stiffness and damping coefficients. Any change larger than a prescribed error bound indicates fault in the system. 


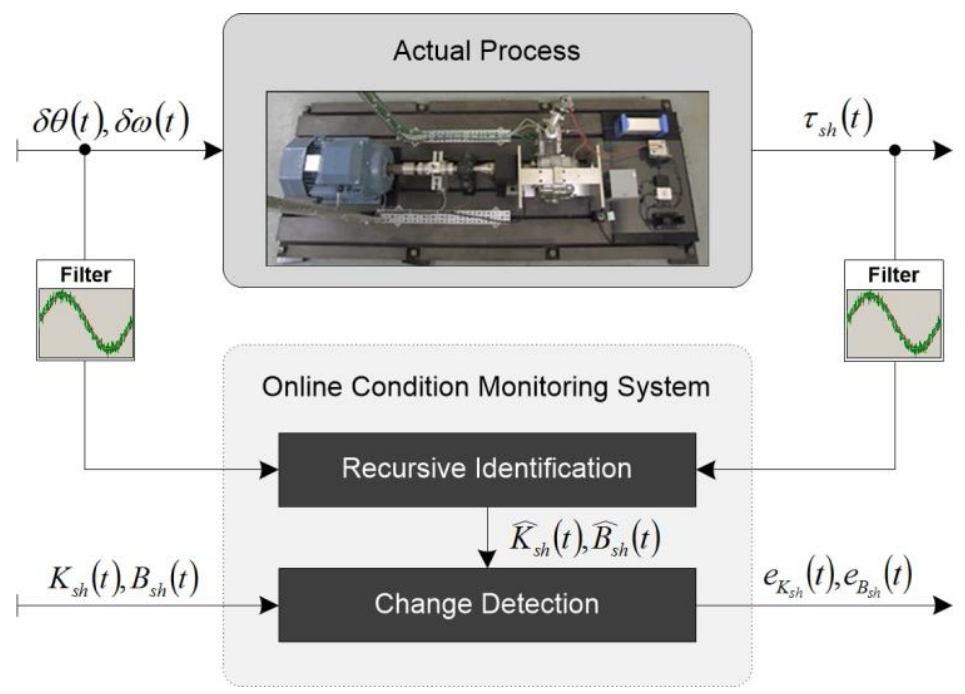

Figure 5: Shaft on-line condition monitoring system, schematic diagram. 


\section{Results and Analysis}

Overall, eight experiments were carried out and used for assessing the current damage detection method's effectiveness, six real world experiments under two shaft speeds and three types of shafts spiders and two simulation experiments under two types of damages. More details and results from the practical implementation of the condition monitoring system are presented below.

\section{Experimental Validation}

The performance of the recursive estimators was tested under different operating conditions to ensure the robustness and repeatability of the health monitoring system. Measurements and results from two different test cases i.e. steady state Fig. 6 and transient Fig. 7 are presented. The main observation data required for the implementation of the coupling shaft condition monitoring system are the velocity Fig. 6a and Fig. 7a and position Fig. $6 \mathrm{c}$ and Fig. 7c of the engine and dynamometer respectively and the torque of the shaft Fig. 6e and Fig. 7e. The testing duration for both the steady-state experiment Fig. 6 and the transient experiment Fig. 7 is 10 seconds in total. As it will be shown later, the time was more than enough for the recursive estimators to converge and start tracking effectively the physical parameters of the coupling shaft. On the other hand, one practical issue that affected the quality of the identified coupling shaft parameters was the measurement of the velocity of the engine and the dynamometer. More specifically, as it can be seen in Fig. $6 \mathrm{~b}$ and Fig. $7 \mathrm{~b}$ the difference between the engine and dynamometer velocity (Eq. 11) does not obey a cyclic behaviour as for example the position difference 
(Eq. 10), see Fig. 6d and Fig. 7d. This observation implies that the velocity measurement was partly problematic and in fact the source of this problem is located in the selection of the time step that is used for the calculation of the velocity; note that the velocity is estimated using the angular position signal i.e. gradient of the position.

(a)

(c)

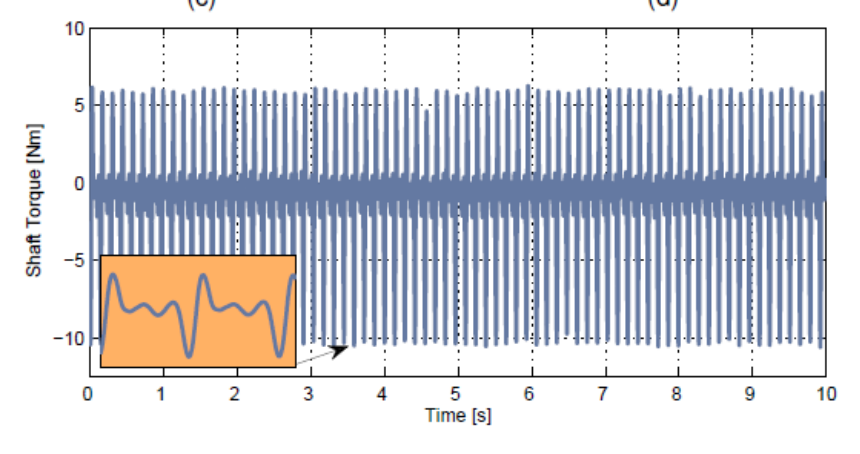

(e)

(b)

(d)
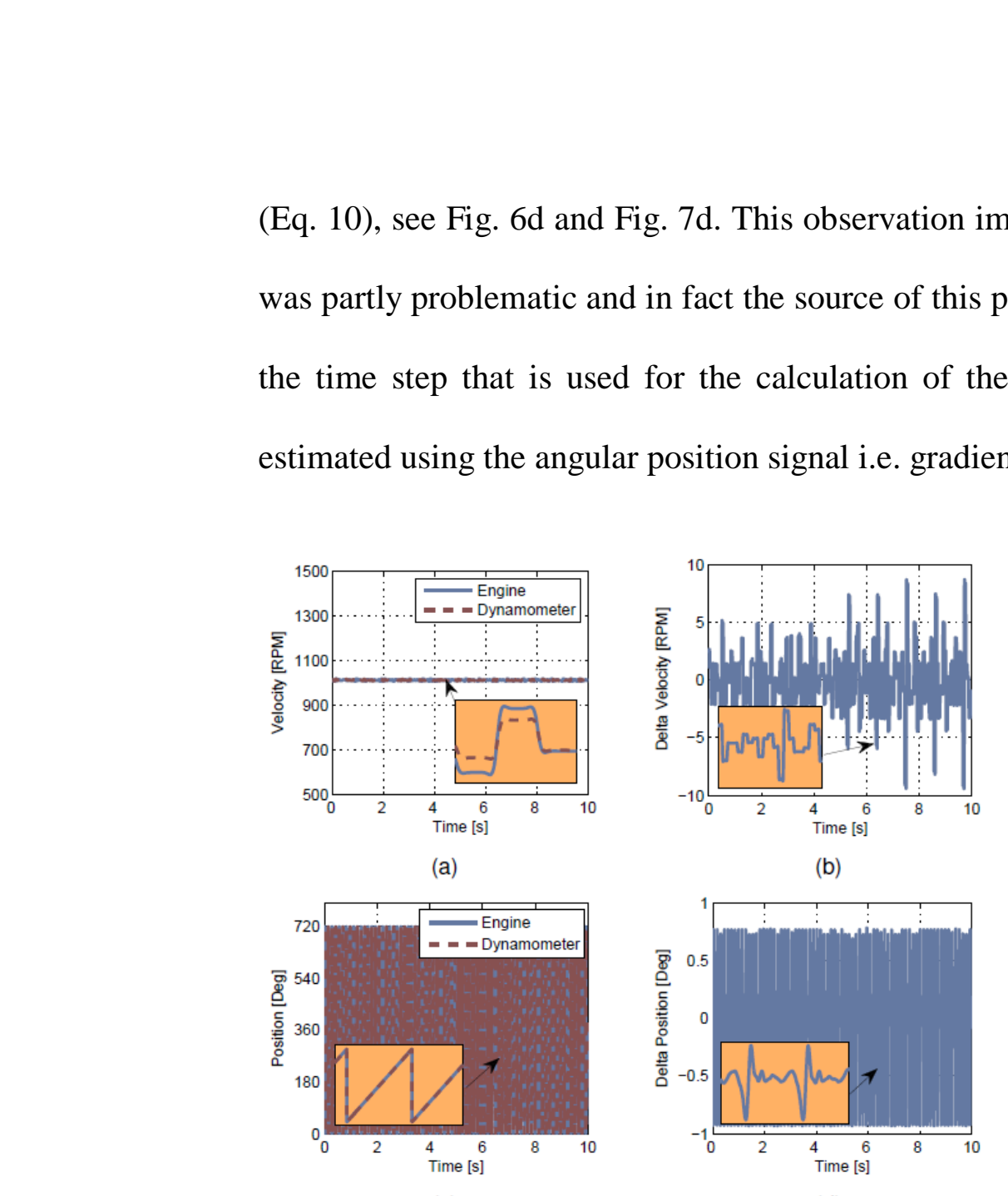

$$
\text { (1) }
$$

Figure 6: Constant speed experiment (Test ID: T01), (a) velocity, (b) velocity difference, (c) cyclic position, (d) position difference, (e) shaft torque. 


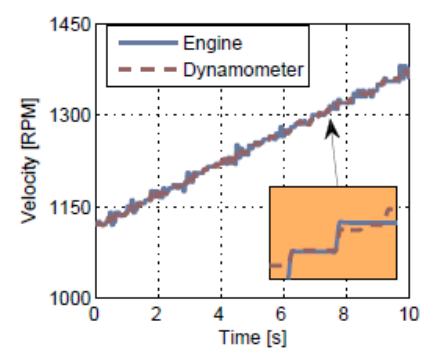

(a)

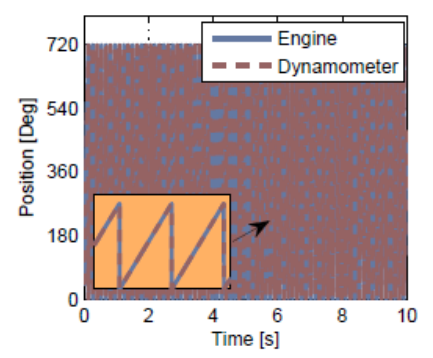

(c)

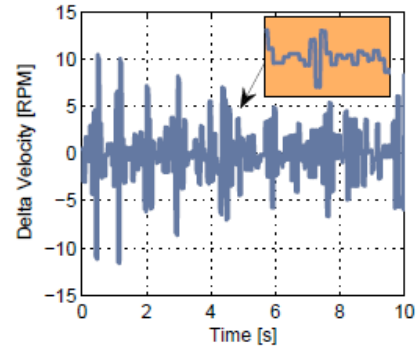

(b)

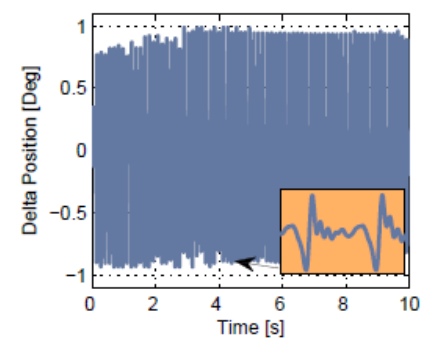

(d)

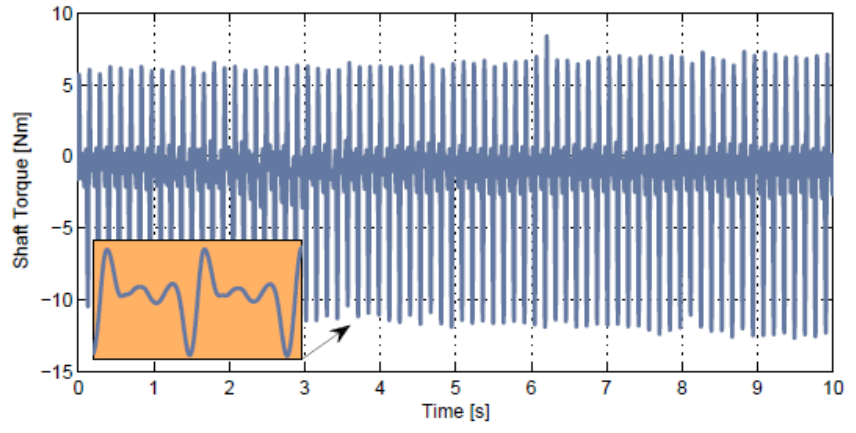

(e)

Figure 7: Speed ramp experiment (Test ID: T02), (a) velocity, (b) velocity difference, (c) cyclic position, (d) position difference, (e) shaft torque. 


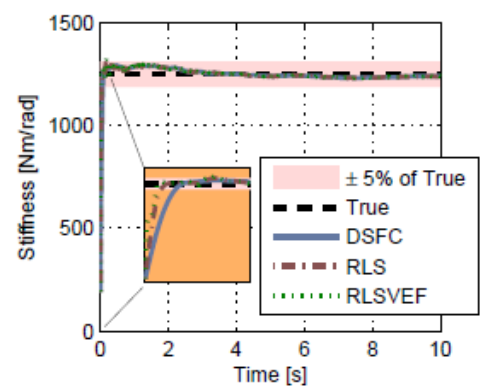

(a)

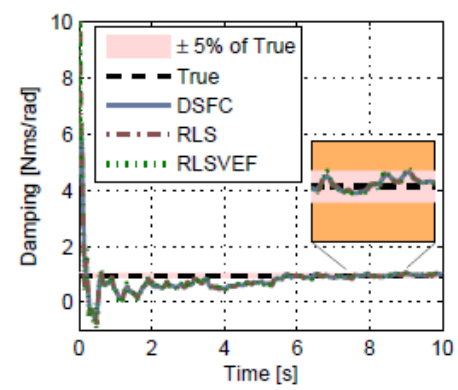

(d)

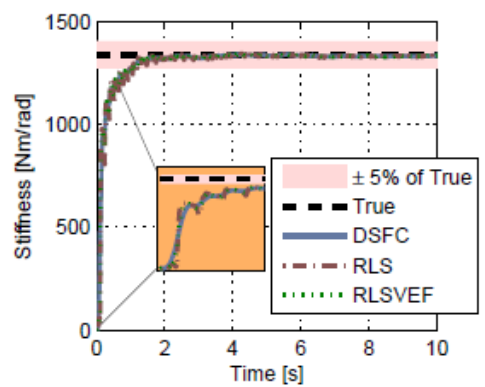

(b)

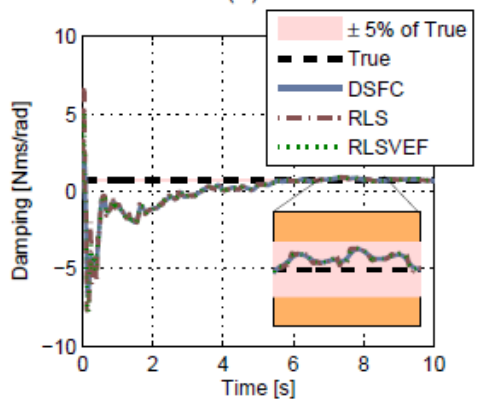

(e)

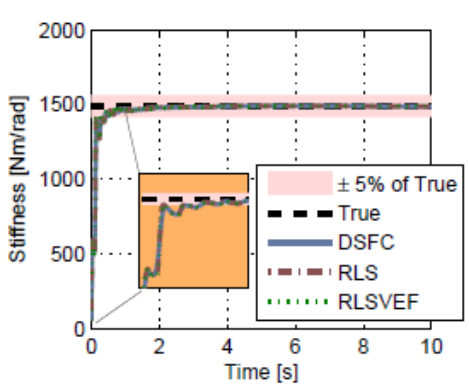

(c)

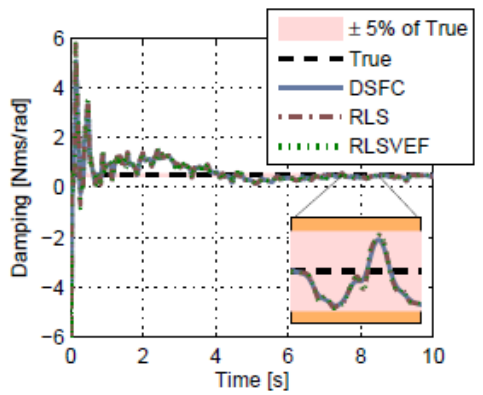

(f)

Figure 8: Estimation results from test T01, (a) stiffness-yellow, (b) stiffness-red, (c) stiffness-green, (d) damping-yellow, (e) damping-red, (f) damping-green. 


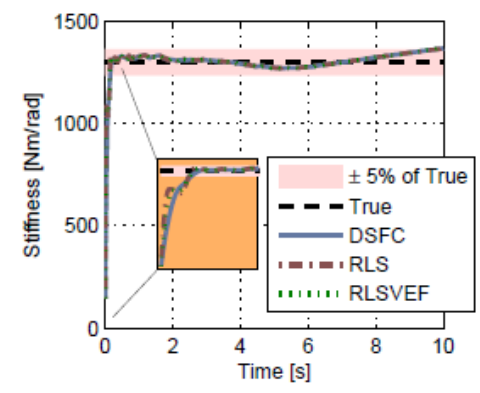

(a)

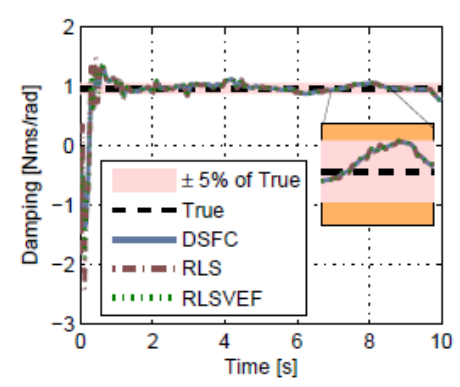

(d)

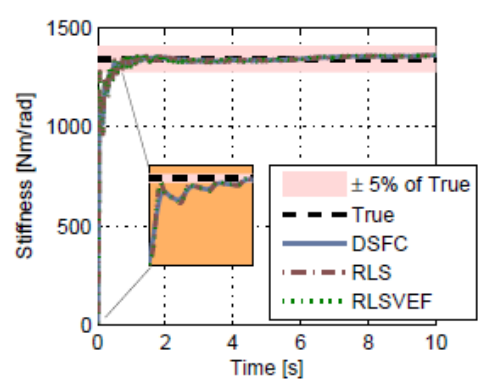

(b)

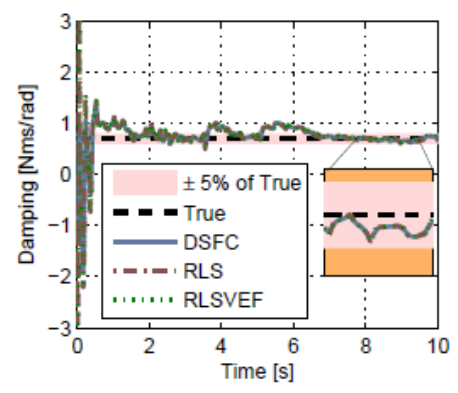

(e)

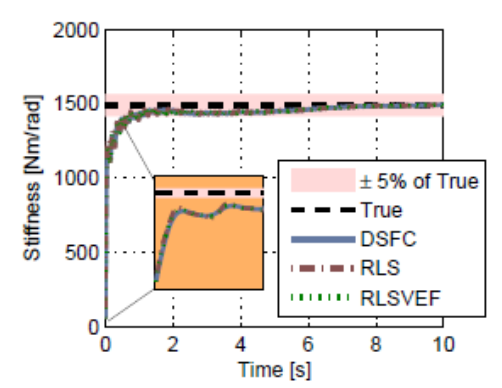

(c)

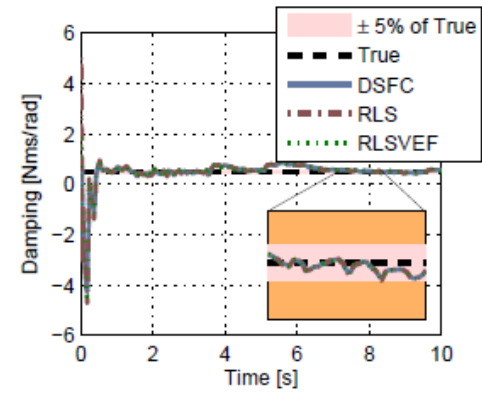

(f)

Figure 9: Estimation results from test T02, (a) stiffness-yellow, (b) stiffness-red, (c) stiffness-green, (d) damping-yellow, (e) damping-red, (f) damping-green.

The results from the implementation of the three different linear recursive estimators i.e. DSFC, RLS, and RLSVEF, for the constant speed experiment (Fig. 6) and the speed ramp experiment (Fig. 7), are presented in Fig. 8 and Fig. 9 respectively. A first look at the results depicts that all of the applied estimators converge and identify within $\pm 5 \%$ of the true parameters of the coupling shaft within the first 5 seconds of the estimation window. As far as the initialisation of the recursive algorithms is concerned, the parameter estimates were set to 0 for all different algorithms (to examine the performance of the estimators under worst case scenarios) while the confidence constant $c$ of the covariance (RLS, RLSVEF) and the square root covariance (DSFC) matrices was set equal to 1000 for all different test cases. It should be mentioned that the adjustment of those values 
could affect the speed of convergence of the estimator. Additionally the forgetting factors were set equal to 0.98 in RLSVEF and DSFC algorithm as slight changes in the parameters were expected. Regarding the repeatability of the estimators in respect to the various operating conditions, it can be said that they manage to identify the true parameters without any significant errors and this verifies the robustness of the proposed methodology. One general trend that was observed thought, is that the parameter estimates of the damping coefficient (Fig. 8d, Fig. 9d, Fig. 8e, Fig. 9d, Fig. 8f, Fig. 9f) seem to be noisier compared to the parameter estimates of the stiffness coefficient (Fig. 8a, Fig. 9a, Fig. 8b, Fig. 9b, Fig. 8c, Fig. 9c). This was caused by the problematic measurement of engine and dynamometer velocities (damping coefficient is proportional to the velocity difference (Eq. 11) while stiffness coefficient to the position difference (Eq. 10)).

A quantitative comparison among the three recursive estimators is presented in Fig. 10. The performance of the estimators is evaluated using a suitable quantitative statistic namely, Normalised Mean Square Error (NMSE). 


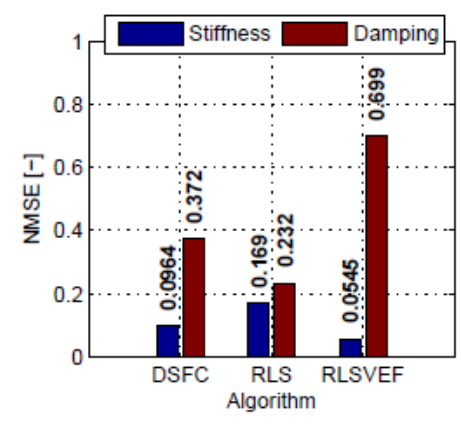

(a)

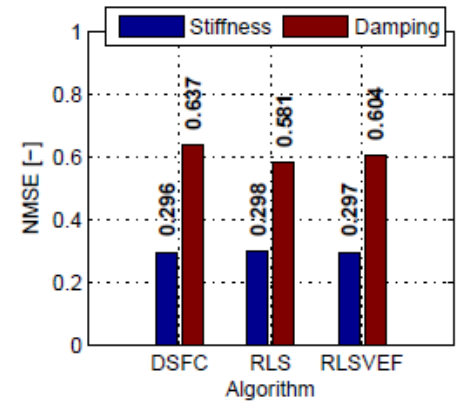

(d)

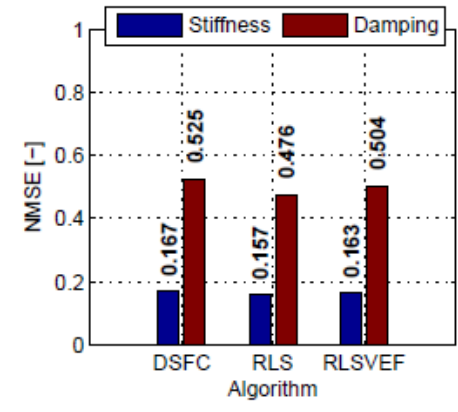

(b)

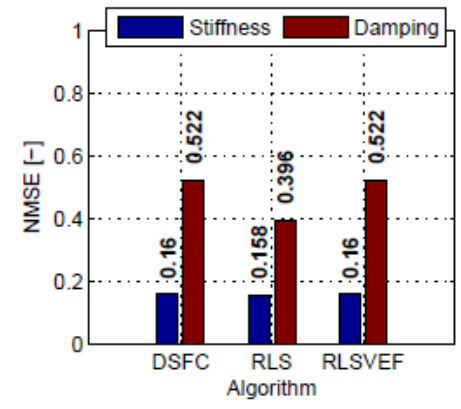

(e)

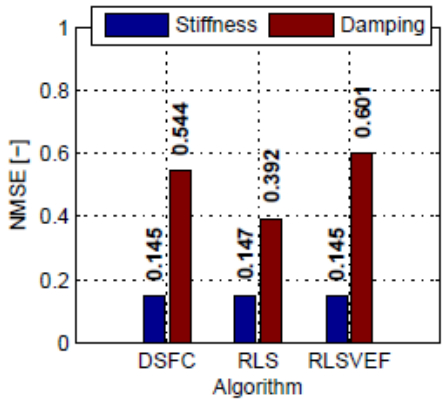

(c)

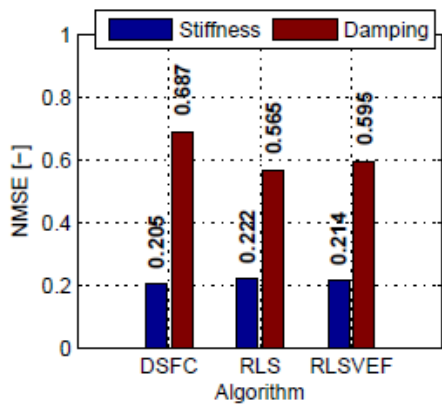

(f)

Figure 10: Quantitative comparison among the three different recursive estimators, and differences between stiffness estimates and damping estimates, (a) yellow spider-Test ID: T01, (b) red spider-Test ID: T01, (c) green spider-Test ID: T01, (d) yellow spider-Test ID: T02, (e) red spider-Test ID: T02, (f) green spider-Test ID: T02.

$$
N M S E=\frac{1}{n} \sum_{i=1}^{n} \frac{\left(Y_{m}-Y_{p}\right)^{2}}{\left(\frac{1}{n} \sum_{i=1}^{n} Y_{m}\right)\left(\frac{1}{n} \sum_{i=1}^{n} Y_{p}\right)}
$$

where, $n$ is the total number of samples, $Y_{m}$ is the measured parameter (true) and $Y_{p}$ is the predicted parameter (estimation). The higher the NMSE the less accurate the corresponding estimation.

According to the statistics presented in Fig. 10, the algorithms estimate the stiffness coefficient with greater accuracy compared to the damping coefficient, in every occasion i.e. operating condition and coupling shaft configuration. This is another 
evidence which support the statement that the problematic velocity measurement affected the coupling shaft parameter estimates. Turning to the estimation accuracy, among the different algorithms, there is no clear evidence that a specific algorithm is superior to another. It is believed that more experimental data would be required in order to be conclusive about the actual performance of the various algorithms. Nonetheless, the main outcome of this comparison reveals that as far as the tracking performance is concerned there is no any significant practical difference between the algorithms. It should be clarified that differences related to the properties of the algorithms such as memory needs and execution time were not investigated as they are beyond the scope of this study.

\section{Fault Simulation}

Given the successful practical implementation of the proposed algorithms, it is attempted to generate some representative coupling shaft fault cases in order to evaluate the effectiveness of the on-line condition monitoring system to track and isolate possible faults. The faults were generated using a simulation model of the complete transient engine test cell, which as presented in (Souflas et al. 2014). Two types of faults were examined, namely, process faults (Fig. 11), and sensor faults (Fig. 12). The process faults involved individual changes in the coupling shaft characteristics i.e. stiffness and damping coefficients, whilst the sensor fault was related to the readings of the dynamometer encoder i.e. change in the measured speed of the dynamometer. 


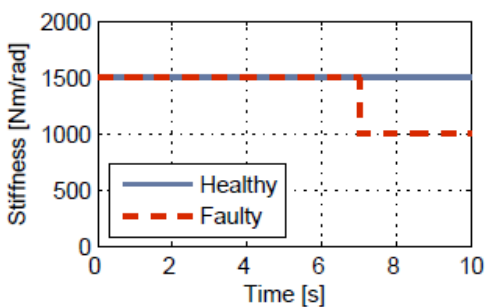

(a)

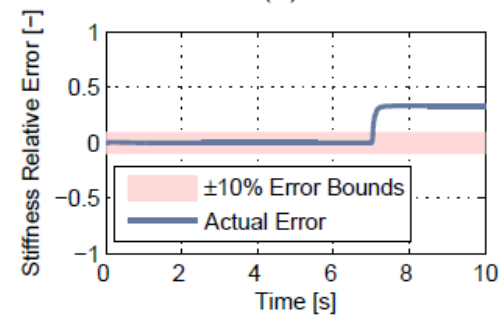

(c)

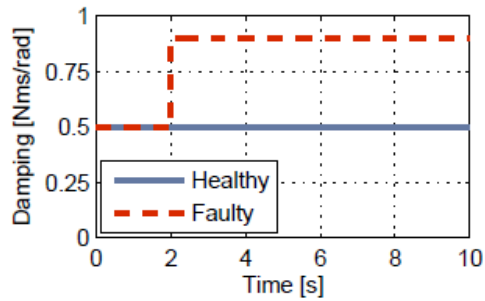

(b)

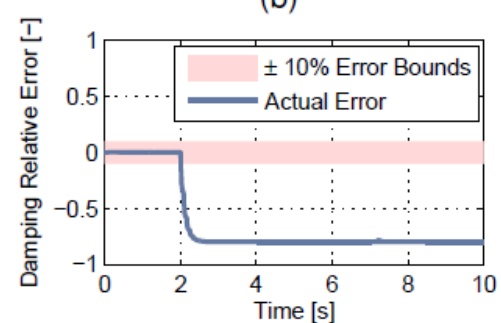

(d)

Figure 11: Process fault simulation, (a) stiffness fault, (b) damping fault, (c) stiffness relative error, (d) damping relative.

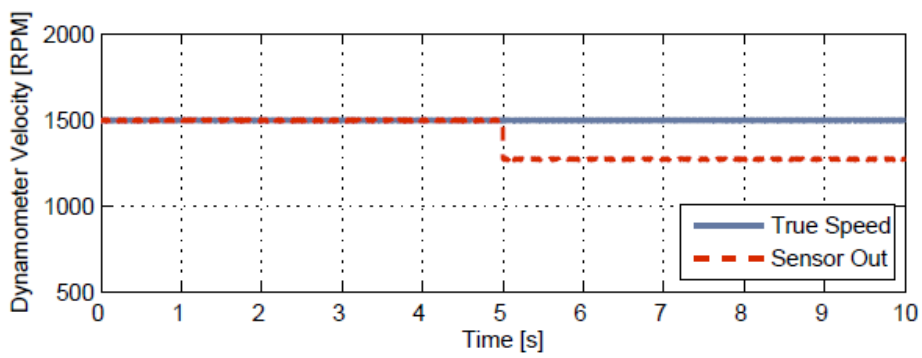

(a)

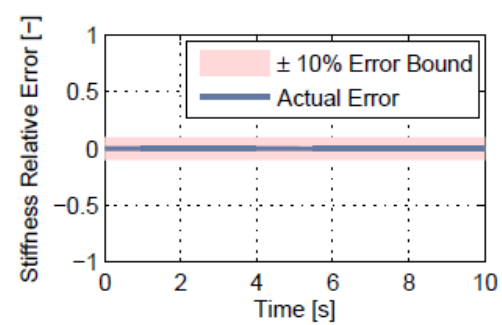

(b)

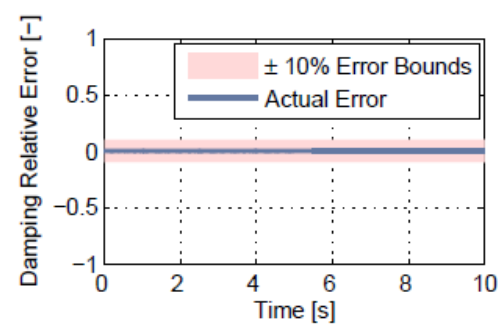

(c)

Figure 12: Sensor fault simulation, (a) speed sensor fault, (b) stiffness relative error, (c) damping relative error. 
For this study the coupling shaft model was parametrised using the estimates of the green spider i.e. $K_{s h} \approx 1490\left[\frac{\mathrm{Nm}}{\mathrm{rad}}\right]$ and $B_{s h} \approx 0.52\left[\frac{\mathrm{Nms}}{\mathrm{rad}}\right]$, whilst only the DSFC algorthm was implemented for tracking any changes in the parameters. The forgetting factor was set to 0.98 , as changes in the parameters were expected. Furthermore the DSFC algorithm was properly initialised (parameter estimates and square root of covariance matrix) as the healthy conditions of the coupling shaft were already known.

The simulation results revealed that the proposed condition monitoring system can effectively isolate different process faults, as when changes in stiffness coefficient (Fig. 11a and Fig. 11c) occur the damping coefficient (Fig. 11b and Fig. 11d) estimate is not affected and vice versa. Similarly in the case of the sensor fault, when the speed sensor of the dynamometer provides wrong readings, the parameter estimates of the coupling shaft are not affected. This is also what was expected as sensor faults should not result in misleading parameter estimates of the coupling shaft. Consequently, it can be said the suggested on-line condition monitoring method can be used to isolate various fault cases whether they are caused due to process or sensor errors. 


\section{Conclusions}

A practically viable health monitoring system for engine dynamometer shafts based on adaptive identification was presented in this paper. The effectiveness of the methodology was demonstrated with real-world experiments on a prototype transient engine test cell but also with additional simulation studies. The results of this work prove that the condition monitoring of engine dynamometer shafts can be achieved using a simple shaft model and a linear recursive identification algorithm. However, it is also highlighted that the accuracy of the parameter estimates was considerably affected by noisy measurements e.g. velocity measurement and damping coefficient estimate.

The tracking performance of three different recursive algorithms namely RLS, RLSVEF, DFCI, was examined and the results revealed that there is no any significant practical difference between the algorithms. Future work could be focused on investigating the properties of the algorithms such as memory needs and execution time to understand if there are any practical difference as far as implementation is concerned.

Lastly, it is useful to mention that this piece of work is particularly useful in applications were the continues condition monitoring of rotating shafts is vital such as automotive and marine propulsions, machine tools and large-scale drilling devices.

\section{Conflict of Interest}

None declared. 


\section{References}

Åström, K.J. \& Eykhoff, P., (1971). System identification-A survey. Automatica, 7(2), pp.123-162. Available at: http://www.sciencedirect.com/science/article/pii/0005109871900598.

Åström, K.J. \& Wittenmark, B., (2013). Adaptive Control: Second Edition, Dover Publications. Available at: https://books.google.co.uk/books?id=4CLCAgAAQBAJ.

Cade, I.S., Keogh, P.S. \& Sahinkaya, M.N., (2005). Fault identification in rotor/magnetic bearing systems using discrete time wavelet coefficients. IEEE/ASME Transactions on Mechatronics, 10(6), pp.648-657.

Calabrese, A., Strano, S. \& Terzo, M., (2016). Parameter estimation method for damage detection in torsionally coupled base-isolated structures. Meccanica, 51(4), pp.785797. Available at: http://link.springer.com/10.1007/s11012-015-0257-2.

Castejón, C. et al., (2015). Automatic detection of cracked rotors combining multiresolution analysis and artificial neural networks. Journal of Vibration and Control , 21 (15), pp.3047-3060. Available at: http://jvc.sagepub.com/content/21/15/3047.abstract.

Chaubey, A., Chelladurai, H. \& Lamba, S.S., (2014). Condition monitoring of rotating shaft using virtual instrumentation. In 5th International and 26th All India Manufacturing Technology, Design and Research Conference (AIMTDR 2014). IIT Guwahati, Assam, India, p. 6.

Hsieh, C.-S., Liaw, D.-C. \& Lin, T.-H., (2013). Optimal Filtering Methods to Structural Damage Estimation under Ground Excitation. The Scientific World Journal, 2013, pp.1-12. Available at: http://www.hindawi.com/journals/tswj/2013/528109/.

Isermann, R. \& Münchhof, M., (2010). Identification of Dynamic Systems: An Introduction with Applications, Springer Berlin Heidelberg. Available at: http://books.google.co.uk/books?id=tTwYA6ph9L0C.

Kaminski, P., Bryson, A.E. \& Schmidt, S., (1971). Discrete square root filtering: A survey of current techniques. Automatic Control, IEEE Transactions on, 16(6), pp.727-736.

Ljung, L., (2002). Recursive identification algorithms. Circuits, Systems and Signal Processing, 21(1), pp.57-68. Available at: http://dx.doi.org/10.1007/BF01211651.

Ljung, L. \& T. Söderström, (1983). Theory and Practice of Recursive Identification A.S. Willsky, ed., Cambridge, Mass., USA: MIT Press. 
Seibold, S. \& Fritzen, C.-P., (1995). Identification Procedures as Tools for Fault Diagnosis of Rotating Machinery. International Journal of Rotating Machinery, 1(34), pp.267-275. Available at: http://www.hindawi.com/journals/ijrm/1995/401837/abs/.

Seibold, S. \& Weinert, K., (1996). A Time Domain Method for the Localization of Cracks in Rotors. Journal of Sound and Vibration, 195(1), pp.57-73. Available at: http://www.sciencedirect.com/science/article/pii/S0022460X96904034.

Sekhar, A.S., (2004). Crack identification in a rotor system: a model-based approach. Journal of Sound and Vibration, 270(4-5), pp.887-902. Available at: http://www.sciencedirect.com/science/article/pii/S0022460X03006370.

Souflas, I. et al., (2014). Dynamic Modeling of a Transient Engine Test Cell for Cold Engine Testing Applications. ASME 2014 International Mechanical Engineering Congress \& Exposition.

Souflas, I., Pezouvanis, A. \& Ebrahimi, K.M., (2015). Nonlinear Recursive Estimation With Estimability Analysis for Physical and Semiphysical Engine Model Parameters. Journal of Dynamic Systems, Measurement, and Control, 138(2), p.024502. Available at:

http://dynamicsystems.asmedigitalcollection.asme.org/article.aspx $?$ doi=10.1115/1.4 032052.

Szabat, K., Orowska-Kowalska, T. \& Serkies, P., (2011). Robust Control of the Twomass Drive System Using Model Predictive Control. In Robust Control, Theory and Applications. InTech. Available at: http://www.intechopen.com/books/robustcontrol-theory-and-applications/robust-control-of-the-two-mass-drive-system-usingmodel-predictive-control.

Tlaisi, A.A. et al., (2012). Crack Detection in Shaft Using Lateral and Torsional Vibration Measurements and Analyses. Mechanical Engineering Research, 2(2), p.52.

Vigild, C.W., Chevalier, A.M.R. \& Hendricks, E., (2000). Avoiding Signal Aliasing in Event Based Engine Control. In SAE Technical Paper 2000-01-0268. Available at: http://papers.sae.org/2000-01-0268/.

Wang, F. et al., (2014). Structural Stiffness Identification Based on the Extended Kalman Filter Research. Abstract and Applied Analysis, 2014, pp.1-8. Available at: http://www.hindawi.com/journals/aaa/2014/103102/.

Xiang, J. et al., (2008). Crack detection in a shaft by combination of wavelet-based elements and genetic algorithm. International Journal of Solids and Structures, 45(17), pp.4782-4795. Available at: http://www.sciencedirect.com/science/article/pii/S0020768308001777. 
Yang, J.N. et al., (2006). An adaptive extended Kalman filter for structural damage identification. Structural Control and Health Monitoring, 13(4), pp.849-867. Available at: http://doi.wiley.com/10.1002/stc.84.

\title{
Appendix 1
}

\author{
Acronyms \\ DOF Degree of Freedom \\ DSFC Discrete Square Root Filtering \\ EKF $\quad$ Extended Kalman Filter \\ FEM Finite Element Model \\ NMSE Normalised Mean Square Error \\ RLS Recursive Least Squares \\ RLSF Recursive Least Squares with Forgetting \\ RLSVEF Recursive Least Squares with Vector Forgetting
}

\section{Symbols}

$\begin{array}{lll}a & \mathrm{rad} / \mathrm{s}^{2} & \text { Angular acceleration } \\ a_{d y} & \mathrm{rad} / \mathrm{s}^{2} & \text { Dynamometer angular acceleration } \\ a_{e n} & \mathrm{rad} / \mathrm{s}^{2} & \text { Engine angular acceleration } \\ \epsilon & - & \text { Observation noise } \\ \Lambda & - & \text { Forgetting factor matrix } \\ \lambda & - & \text { Forgetting factor } \\ \omega & \mathrm{rad} / \mathrm{s} & \text { Angular velocity }\end{array}$




\begin{tabular}{|c|c|c|}
\hline$\omega_{d y}$ & $\mathrm{rad} / \mathrm{s}$ & Dynamometer angular velocity \\
\hline$\omega_{e n}$ & $\mathrm{rad} / \mathrm{s}$ & Engine angular velocity \\
\hline$\psi, \gamma, g, f$ & - & DSFC variables \\
\hline$\tau$ & $\mathrm{Nm}$ & Torque \\
\hline$\tau_{d y}$ & $\mathrm{Nm}$ & Dynamometer torque \\
\hline$\tau_{e n}$ & $\mathrm{Nm}$ & Engine torque \\
\hline$\tau_{s h}$ & $\mathrm{Nm}$ & Shaft torque \\
\hline$\vartheta$ & - & System parameter(s) \\
\hline$\hat{\vartheta}$ & - & Parameter estimates \\
\hline$\theta$ & $\mathrm{rad}$ & Angular position \\
\hline$\theta_{d y}$ & $\mathrm{rad}$ & Dynamometer angular position \\
\hline$\theta_{e n}$ & $\mathrm{rad}$ & Engine angular position \\
\hline$\varphi$ & - & Vector of observation variables \\
\hline$A, B, C$ & - & State space matrices \\
\hline$B_{s h}$ & $\mathrm{Nms} / \mathrm{rad}$ & Shaft damping coefficient \\
\hline$c$ & - & Initial estimate confidence constant \\
\hline$e$ & - & Prediction error \\
\hline$I$ & - & Identity matrix \\
\hline$J$ & $\mathrm{kgm}^{2}$ & Inertia \\
\hline$J_{d y}$ & $\mathrm{kgm}^{2}$ & Dynamometer inertia \\
\hline$J_{e n}$ & $\mathrm{kgm}^{2}$ & Engine inertia \\
\hline K & - & Kalman gain \\
\hline
\end{tabular}




$\begin{array}{lll}K_{s h} & \mathrm{Nm} / \mathrm{rad} & \text { Shaft stiffness coefficient } \\ n & - & \text { Number of samples } \\ p_{t} & - & \text { Number of parameters } \\ R_{1} & - & \text { Observation covariance matrix } \\ R_{2} & - & \text { Parameter covariance matrix } \\ S & - & \text { Square root of covariance matrix } \\ t & - & \text { Time } \\ u & - & \text { System input(s) } \\ x & - & \text { System state(s) } \\ y & - & \text { System output(s) } \\ Y_{m} & - & \text { Measured response/parameter } \\ Y_{p} & - & \text { Predicted response/parameter }\end{array}$

\section{Appendix 2}

Table 1: True coupling shaft parameters.

\begin{tabular}{|l|c|c|c|}
\hline \multirow{2}{*}{ Parameter } & \multicolumn{3}{|c|}{ Spider Colour } \\
\cline { 2 - 4 } & Yellow & Red & Green \\
\hline Stiffness $\left[\frac{\mathrm{Nm}}{\mathrm{rad}}\right]$ & 1250 & 1340 & 1490 \\
\hline Damping $\left[\frac{\mathrm{Nms}}{\mathrm{rad}}\right]$ & 0.95 & 0.70 & 0.52 \\
\hline
\end{tabular}

